\title{
Improvement of a Traffic System using Image and Video Processing
}

\author{
Md. Rifat Rayhan ${ }^{1}$, Faysal, Mohammad ${ }^{2}$, Md. Taslim Reza ${ }^{3}$ \\ ${ }^{1,2}$ (EEE, American International University-Bangladesh, Bangladesh) \\ ${ }^{3}$ (EEE, Islamic University of Technology (IUT), Bangladesh)
}

\begin{abstract}
At present, the importance of improving a traffic system has become more important than ever before due to the immense number of vehicles on the road. In this paper a unifying philosophy for carrying out different level of image processing has been presented in order to find the best possible outcomes to solve the vision problem at night time. The whole process was done using the low level and mid level image processing algorithms. As there is no fixed method of doing this kind of transformations, in this paper different types of transformations have used to find out the best possible output. There are different types of edge detection techniques in order to detect the substances properly. In case of segmentation, there are different algorithms which can be manipulated according to the purpose. Here we have used some of these techniques to find out the best possible method for this job. Merging with logarithmic and power law transformation this edge detection and segmentation technique produces the output that we have longed for.
\end{abstract}

Keyword - Image and Video processing, Image Enhancement, Gray Level Transformation, Image Segmentation.

\section{INTRODUCTION}

Millions of Car Accidents occur worldwide each year, and the death toll from this accident in the millions. According to the Traffic Report by the WHO, road traffic accidents kill more than 1.2 million people each year, and about 50 million people are injured or disabled. Projections indicate that these figures will increase by about $65 \%$ over the next 20 years unless there is new commitment to prevention [1] [2]. Efforts are being given to improve the stability of the traffic system by introducing new technologies. Among them image processing is one of the newest technologies introduced to the traffic system.

Humans have historically relied on their vision for tasks ranging from basic instinctive survival skills to detailed and elaborate analysis of works of art. Our ability to guide our actions and engage our cognitive abilities based on visual input is a remarkable trait of the human species, and much of how exactly we do what we do and seem to do it so well remains to be discovered. The ability to see and recognize things has limited functions for human eyes. As a result the sometimes the image itself is needed to be enhanced to make it clearer and take it to the level of human eye. In order to do this the field of image processing has been introduced to the modern world.

The field of image processing has grown considerably during the past decade with the increased utilization of imagery in myriad applications coupled with improvements in the size, speed and cost effectiveness of digital computers and related signal processing technologies. Fields which traditionally used analogue imaging are now switching to digital systems, for their flexibility and affordability. Important examples are medicine, image and video production, photography, remote sensing, and security monitoring. These and other sources produce huge volumes of digital image data every day, more than could ever be examined manually. Digital image processing is concerned primarily with extracting useful information from images. Ideally, this is done by computers, with little or no human intervention. Now if the manipulations are done for a video file then it would become video processing. Video processing uses hardware, software, and combinations of the two for editing the images and sound recorded in video files. Extensive algorithms in the processing software and the peripheral equipment allow the user to perform editing functions using various filters. The desired effects can be produced by editing frame by frame or in larger batches [3].

\section{OBJECTIVES}

The costs of all the road accidents throughout the world add up to a shocking 1-3\% of the world's Gross National Product [4]. Most of the accidents in highways are caused due to lack of precision drivers have for deficient vision. Sometimes it becomes very difficult to identify different objects. At the night it becomes even worse. To improve the situation we are intend to introduce new technologies to the system. For the purpose we will be using image and video processing technology by setting up a monitor in front of the driver 
and provide a proper view to him. The input videos will be processed in the processor and give an output which will be clear enough to the driver. The view in the monitor will give him a proper guideline which will help him to make proper decision by judging the condition. This will make the transport system secured by reducing accidents. As a result the main objective will be to use different image processing techniques to manipulate the image and make it clearer to the human eye.

\section{THEORETICAL ANALYSIS}

An image may be defined as a two-dimensional function, $f(x, y)$, where $\mathrm{x}$ and $\mathrm{y}$ are spatial (plane) coordinates, and the amplitude of $f$ at any pair of coordinates $(x, y)$ is called the intensity or gray level of the image at that point. When $(x, y)$, and the amplitude values of $f$ are all finite, discrete quantities, we call the image a digital image. The field of digital image processing refers to processing digital images by means of a digital computer. A digital image is composed of a finite number of elements, each of which has a particular location and value [5].

There are no clear-cut boundaries in the continuum from image processing at one end to computer vision at the other. However, one useful paradigm is to consider three types of computerized processes in this continuum: low-, mid-, and high-level processes. Low-level processes involve primitive operations such as image preprocessing to reduce noise, contrast enhancement, and image sharpening. A low-level process is characterized by the fact that both its inputs and outputs are images. Mid-level processing on images involves tasks such as segmentation (partitioning an image into regions or objects), description of those objects to reduce them to a form suitable for computer processing, and classification (recognition) of individual objects. A midlevel process is characterized by the fact that its inputs generally are images, but its outputs are attributes extracted from those images (e.g., edges, contours, and the identity of individual objects). Finally, higher-level processing involves "making sense" of an ensemble of recognized objects, as in image analysis, and, at the far end of the continuum, performing the cognitive functions normally associated with vision [5].

Among many sectors of image processing, 'image improvement' has received much interest with the publicity given to applications in space imagery and medical research. Here in this paper an attempt of using image improvement for transport system was taken.

\subsection{Image Enhancement}

Image enhancement processes consist of a collection of techniques that seek to improve the visual appearance of an image or to convert the image to a form better suited for analysis by a human or a machine. The enhancement operations are performed in order to modify the image brightness, contrast or the distribution of the gray levels. As a consequence the pixel value (intensities) of the output image will be modified according to the transformation function applied on the input values. Image enhancement simply means, transforming an Image $\mathrm{f}$ into image $\mathrm{g}$ using $\mathrm{T}$. (Where $\mathrm{T}$ is the transformation). The values of pixels in images $\mathrm{f}$ and $\mathrm{g}$ are denoted by $\mathrm{r}$ and $\mathrm{s}$, respectively. As said, the pixel values ' $\mathrm{r}$ ' and ' $\mathrm{s}$ ' are related by the expression,

$$
\mathrm{s}=\mathrm{T}(\mathrm{r})
$$
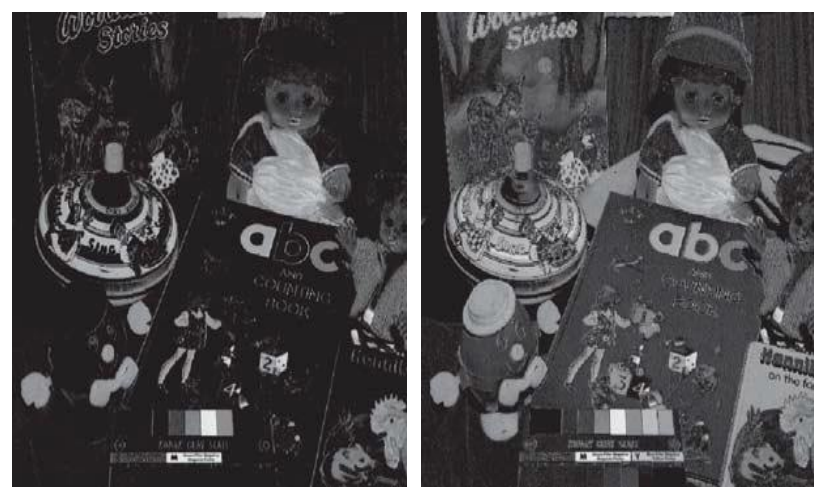

Fig 1: Example of image enhancement

\subsection{Used Gray Level Transformations}

Gray level transformations are the most widely used image enhancement techniques. There are many Gray level transformations and among them some of them were used in this work. The simplest spatial domain operations occur when the neighbourhood is simply the pixel itself. In this case $\mathrm{T}$ is referred to as a grey level transformation function or a point processing operation. Point processing operations take the form $s=T(r)$ 


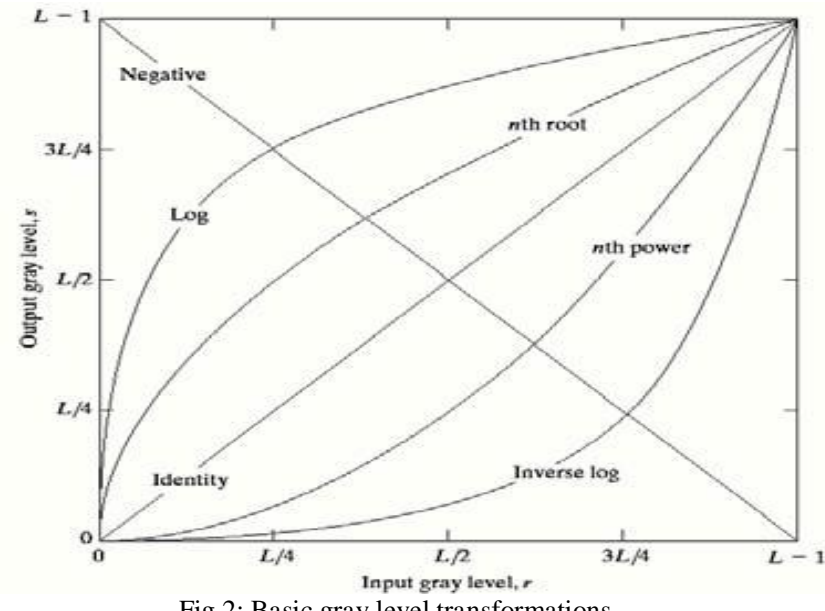

3.2.1 Contrast Manipulation: One of the most common defects of photographic or electronic images is poor contrast resulting from a reduced, and perhaps nonlinear, image amplitude range.

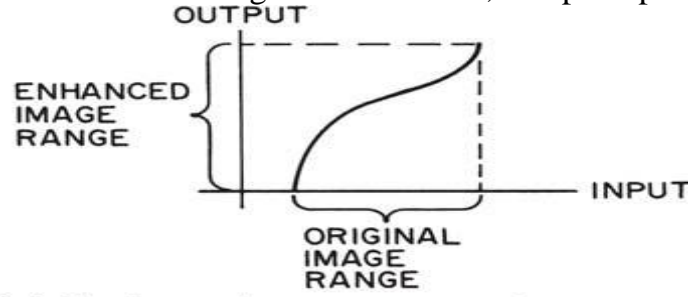

(a) Continuous image contrast enhancement
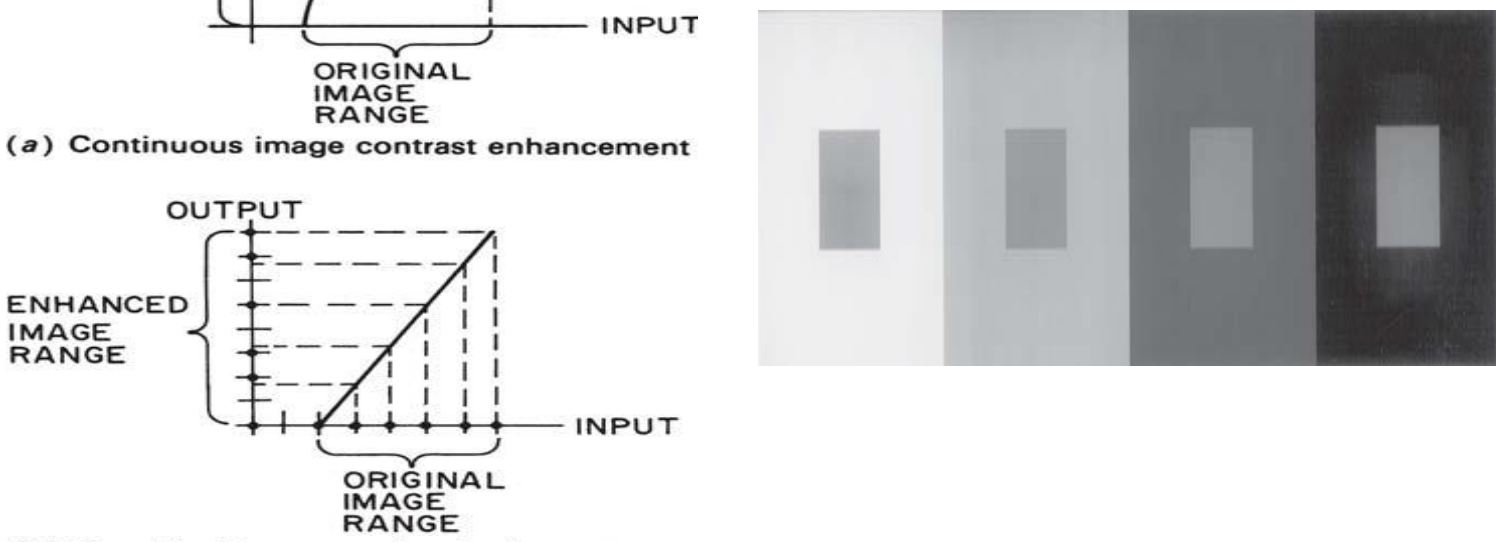

(b) Quantized image contrast enhancement

Fig.3: Continuous and quantized image contrast enhancement

Fig.4: Simultaneous contrast manipulation.

3.2.2 Logarithmic Transformations: Because digital images are displayed as a discrete set of intensities, the eye's ability to discriminate between different intensity levels is an important consideration in presenting imageprocessing results. The ranges of light intensity levels to which the human visual system can adapt is enormous - on the order of $10^{10}$ - from the scotopic threshold to the glare limit [6]. Experimental evidence indicates that subjective brightness (intensity as perceived by the human visual system) is a logarithmic function of the light intensity incident on the eye.

The log transformation maps a narrow range of low input grey level values into a wider range of output values. The inverse log transformation per forms the opposite transformation. Log functions are particularly useful when the input grey level values may have an extremely large range of values. In the following example the Fourier transform of an image is put through a log transform to reveal more details [7].

The general form of the log transformation is

$$
\mathrm{S}(\mathrm{i}, \mathrm{j})=\mathrm{c} \times \log |\mathrm{r}(\mathrm{i}, \mathrm{j})|
$$

Since the logarithm is not defined for 0 , many implementations of this operator add the value 1 to the image before taking the logarithm. The operator is then defined as

$$
\mathrm{S}=\mathrm{c} \times \log (1+\mathrm{r})
$$



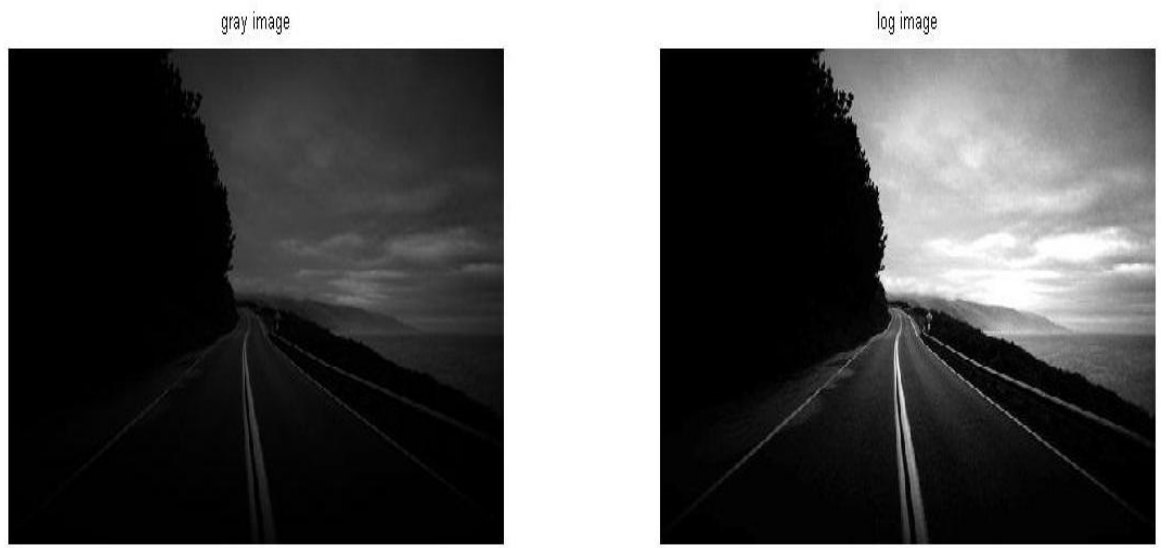

Fig.5: comparison between gray and logarithmic transformed image with $\mathrm{c}=2$

3.2.3 Power-Law Transformations: The nth power and nth root curves shown in fig.6 can be given by the expression,

$$
s=c r^{\gamma}
$$

Where $\mathrm{r}$ is the original pixel value, $\mathrm{s}$ is the resulting pixel value, $\mathrm{c}$ is a scaling constant, and $\gamma$ is a positive value. Figure 6 shows a plot of equation (4) for several values of $\gamma$. This transformation function is also called as gamma correction. For various values of $\gamma$ different levels of enhancements can be obtained [8].

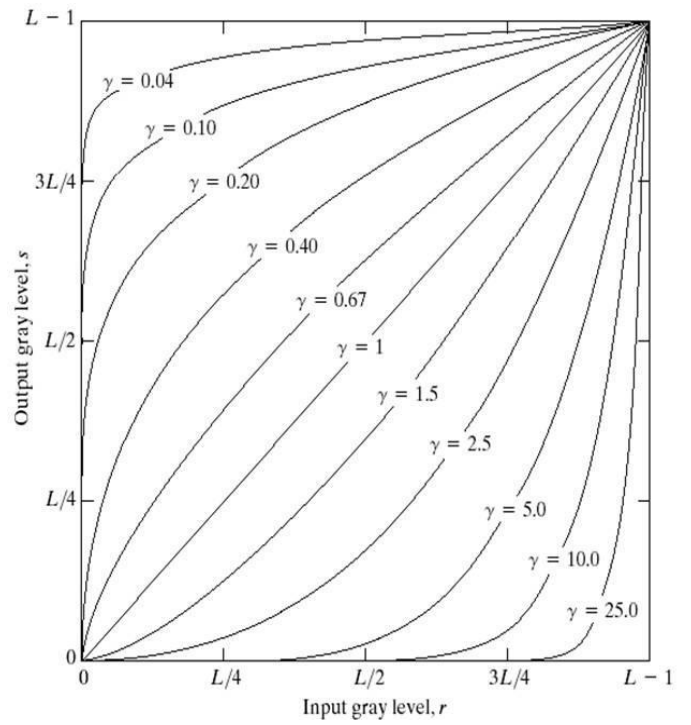

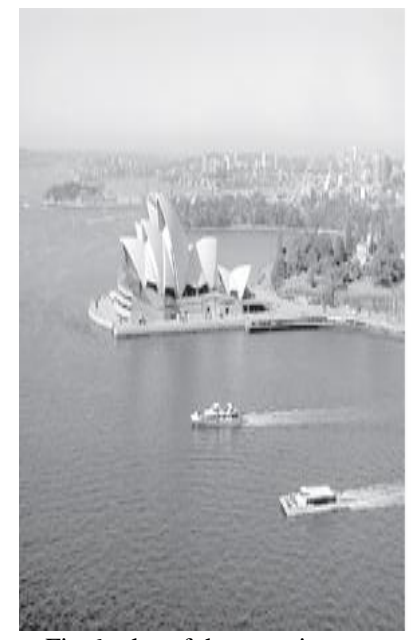

Fig 6: plot of the equation $s=c r^{\gamma}$

\subsection{Image Segmentation:}

"As there is no theory of clustering, there is no theory of image segmentation. Image segmentation techniques are basically ad hoc and differ precisely in the way they emphasize one or more of the desired properties and in the way they balance and compromise one desired property against another." --Haralick and Shapiro

The main goal of image segmentation is domain independent partitioning of an image into a set of disjoint regions that are visually different, homogeneous and meaningful with respect to some characteristics or computed property(ies), such as grey level, texture or colour to enable easy image analysis (object identification, classification and processing)

In image processing, the expression 'Image segmentation' means that given a function $f: E \rightarrow T$, where the space $E$ is partitioned into zones in which the function $f$ is homogeneous, in some optimal sense. There are essentially two ways for thinking this optimization problem. One of them associates 'numbers' with partitions and makes the optimization to hold on these numbers. The first way, said to be variational [9], associates the image with an energy which represents the amount of information left in each smooth version of image. The second way acts directly on the set of all partitions and expresses optimization in this partition space. 
Discontinuity and similarity/homogeneity are two basic properties of the pixels in relation to their local neighbourhood used in many segmentation methods. The segmentation methods that are based on discontinuity property of pixels are considered as boundary or edges based techniques and that are based on similarity or homogeneity are region based techniques.

It must noted that so many image segmentation surveys have been conducted but there very few who have presented how researchers can evaluate one's technique against the other on a domain independent images or evaluate the performance of their segmentation $[10,11]$.

Many surveys have been directed to one area of application of image segmentation in areas such as medical, remote sensing and image retrieval [12]. But here in this paper we have tried to find an optimized segmentation technique for night time road segmentation for the traffic system.

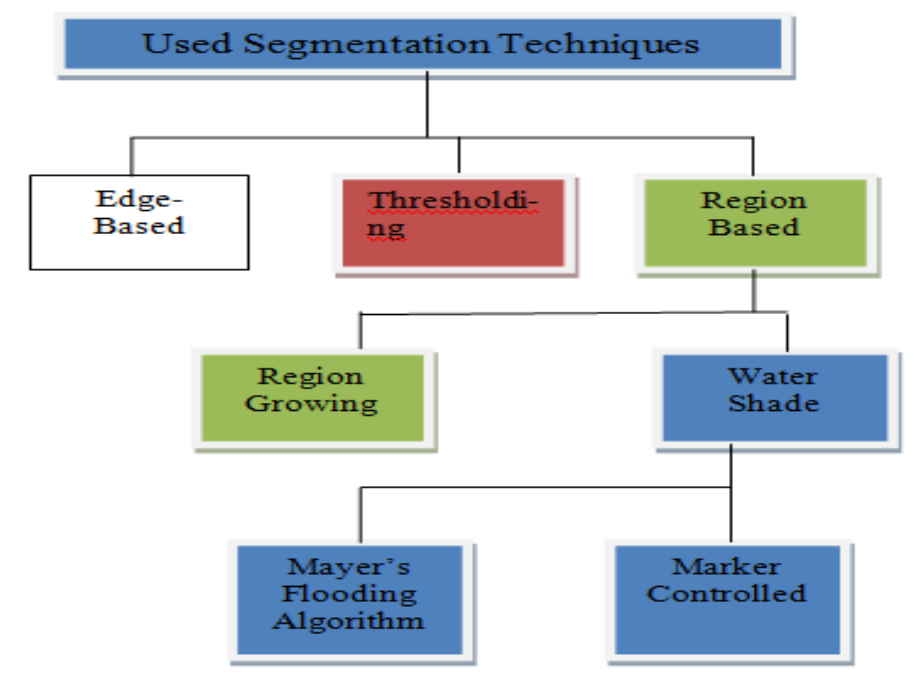

Fig.8 indicates the classification of image segmentation techniques those were examined in this work.

\subsubsection{Edge Based Methods}

Edge based segmentation is the location of pixels in the image that correspond to the boundaries of the objects seen in the image. It is then assumed that since it is a boundary of a region or an object then it is closed and that the number of objects of interest is equal to the number of boundaries in an image. For precision of the segmentation, the perimeter of the boundaries detected must be approximately equal to that of the object in the input image. In the endeavour to implement the above there was need to define an edge in an image. An edge or a linear feature is manifested as an abrupt change or a discontinuity in digital number of pixels along a certain direction in an image. The manifestation becomes a high gradient/ extreme of first order derivative or a zero crossing in the second derivatives.

This brought another assumption that every object of interest in an image has a boundary that can be detected through the use of gradient or second derivative. Sobel, Prewitt and watershed just to mention a few use templates based on gradient/first derivative to detect the boundaries of an image. The Laplacian template is based on second derivative. These methods in general can be defined by function $\mathrm{g}(\mathrm{x})$ that will act as stopping term when the object/region boundary has been reached. A function $\mathrm{g}(\mathrm{x})$ can be defined as

$$
\mathrm{g}(\mathrm{Z}) \geq 0 \text { and } \lim _{z \rightarrow \infty} g(Z)=0
$$

For instance

$$
g(|\nabla u(x, y)|)=\frac{1}{1+|\nabla G(x, y) \times u(x, y)|^{p}}
$$

Where, $\mathrm{p} \geq 1$, and $G \times u$, is the convolution of the image $\mathrm{u}$, with the Gaussian filter $G(x, y)$ which results in a smoother version of image $u$. But there are problems that have been areas of interest for researchers and the problems are centered on the use of gradient to detect the boundaries [13].

For instance, these methods have problems with images that are:

1. Edge-less

2. Very noisy

3. Boundary that is very smooth

4. Texture boundary

Other problems of these techniques emanate from the failure to adjust/calibrate gradient function accordingly thus produces undesirable results as: 
1. The segmented region might be smaller or larger than the actual

2. The edges of the segmented region might not be connected

3. Over or under-segmentation of the image (arising of pseudo edges or missing edges)

\subsubsection{Thresholding Method:}

Thresholding based image segmentation aims to partition an input image into pixels of two or more values through comparison of pixel values with the predefined threshold value $\mathrm{T}$ individually;

Let I $(i, j)$ be an image, then

$$
I(i, j)=\left\{\begin{array}{l}
0, p(i, j)<T \\
1, p(i, j) \geq T
\end{array}\right.
$$

Where $p(i, j)$ refers to the pixel value at the position $(i, j)$. Thresholding may be implemented locally or globally. In global thresholding the image is partitioned into two as shown above while local thresholding the image is subdivided into sub images and the threshold for each sub image is derived from the local properties of the pixels. The predefined value of $\mathrm{T}$ is the one that complicates this method. The determination of the value $\mathrm{T}$ has been the point of interest in image segmentation research [14].There have been many algorithms developed to generate better threshold value $\mathrm{T}$ to segment an image. [15]

These methods that use intensity value do not use spatial morphological image information of an image and they usually fail to segment objects with low contrast or noisy images with varying background. [16]

Failure to find the most suitable algorithm to determine the threshold value(s) $\mathrm{T}$ the result might be one or all of the following:

1. The segmented region might be smaller or larger than the actual

2. The edges of the segmented region might not be connected

3. Over or under-segmentation of the image (arising of pseudo edges or missing edges)

\subsubsection{Water Shade Segmentation:}

Watershed segmentation is a way of automatically separating or cutting apart particles that touch. It starts with a mask or binary image, where the particles are (say) black. It calculates a distance map to find the fattest parts of the object (the peaks or local maxima of the distance map). Starting with the peaks as maximal erosion points (MEP's), it dilates them as far as possible - either until the edge of the object is reached, or the edge of the region of another (growing) MEP.

Separating touching objects in an image is one of the most difficult image processing operations. The watershed transform is often applied to this problem. The watershed transform finds "catchment basins" and "watershed ridge lines" in an image by treating it as a surface where light pixels are high and dark pixels are low.

A grey-level image may be seen as a topographic relief, where the grey level of a pixel is interpreted as its altitude in the relief. A drop of water falling on a topographic relief flows along a path to finally reach a local minimum. Intuitively, the watershed of a relief corresponds to the limits of the adjacent catchment basins of the drops of water.

In image processing, different watershed lines may be computed. In graphs, some may be defined on the nodes, on the edges, or hybrid lines on both nodes and edges. Watersheds may also be defined in the continuous domain. There are also many different algorithms to compute watersheds.

The watershed transform can be classified as a region-based segmentation approach. The intuitive idea underlying this method comes from geography: it is that of a landscape or topographic relief which is flooded by water, watersheds being the divide lines of the domains of attraction of rain falling over the region [17]. An alternative approach is to imagine the landscape being immersed in a lake, with holes pierced in local minima. Basins (also called 'catchment basins') will fill up with water starting at these local minima, and, at points where water coming from different basins would meet, dams are built. When the water level has reached the highest peak in the landscape, the process is stopped. As a result, the landscape is partitioned into regions or basins separated by dams, called watershed lines or simply watersheds. When simulating this process for image segmentation, two approaches may be used: either one first finds basins, then watersheds by taking a set complement; or one computes a complete partition of the image into basins, and subsequently finds the watersheds by boundary detection.

\section{RESULTS}

The field of image processing is very complicated to work with as the surrounding changes with time. For that reason a number of techniques have been shown here to get the best result. 
4.1 Logarithmic Transformation:

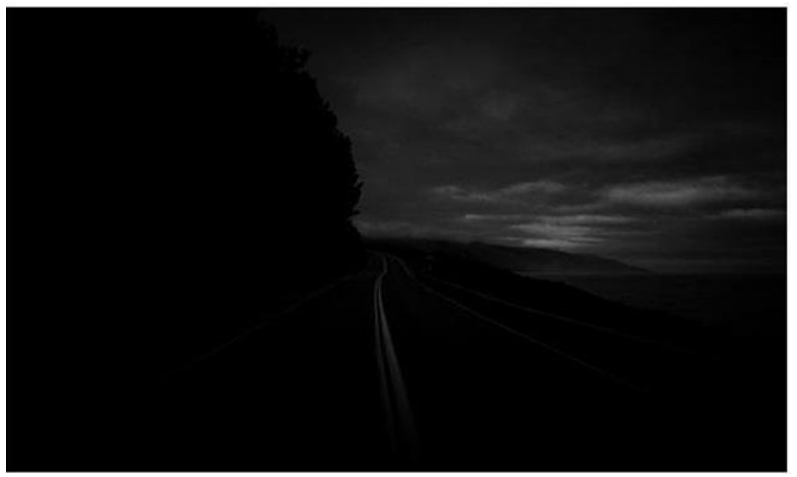

Fig 9: Gray level transformation

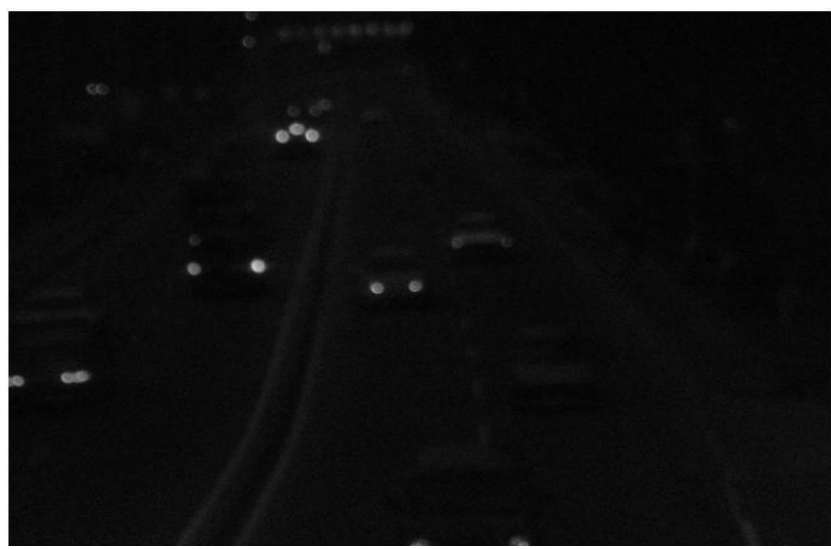

Fig 11: Gray Image

\subsection{Power Law Transformation:}

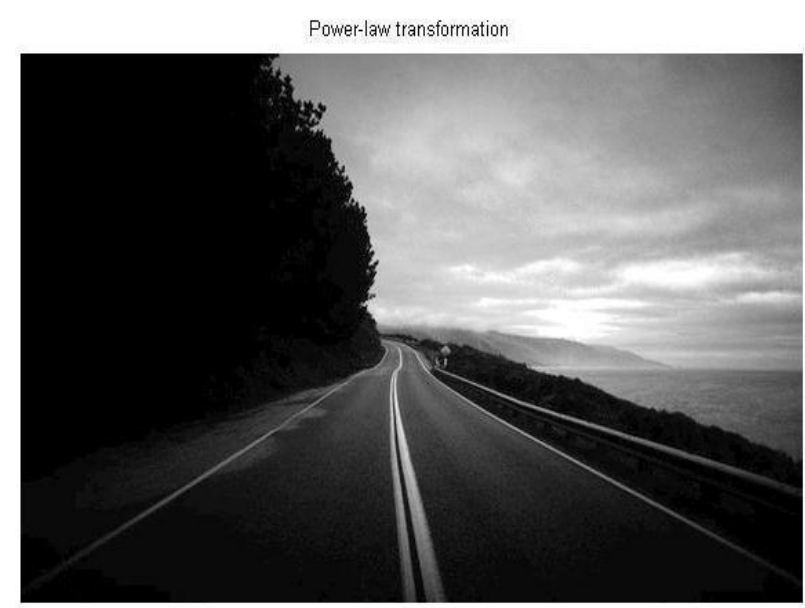

0.7

Fig 13: Power Law Transform with gamma $=0.7$

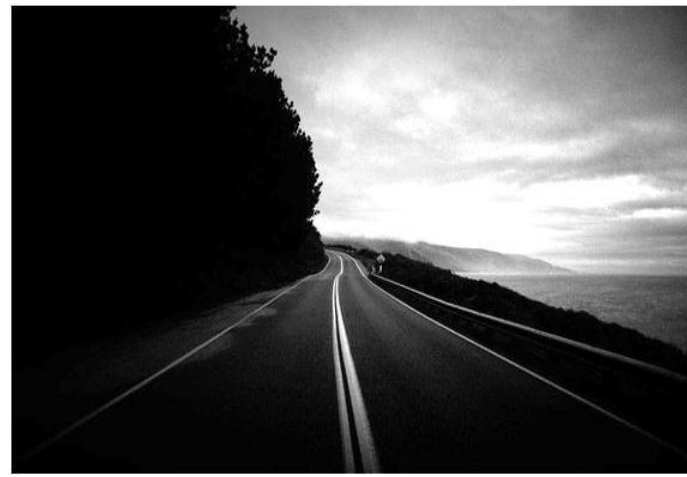

Fig 10: Log image of figure 9 with coefficient 4

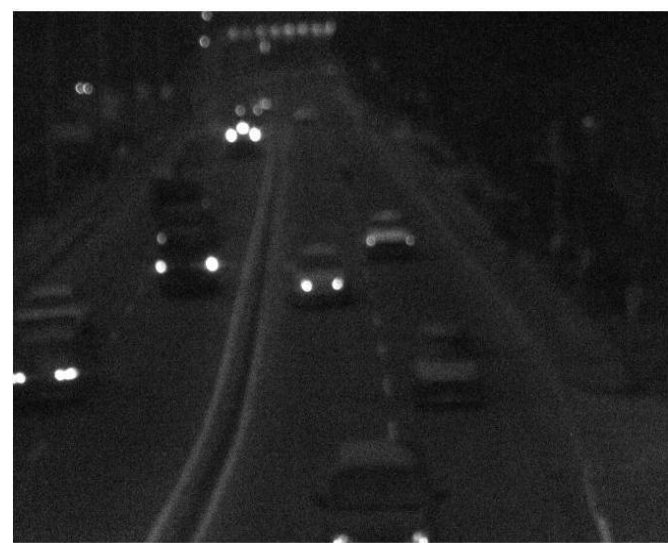

Fig 12: Log image of figure 11 with coefficient 3

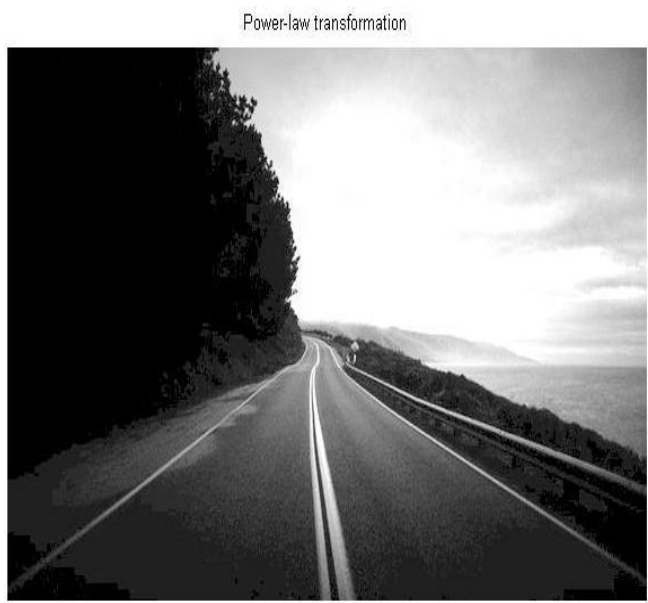

0.5

Fig 14: Power Law Transform with gamma $=0.5$ 
4.3 Region Based segmentation:

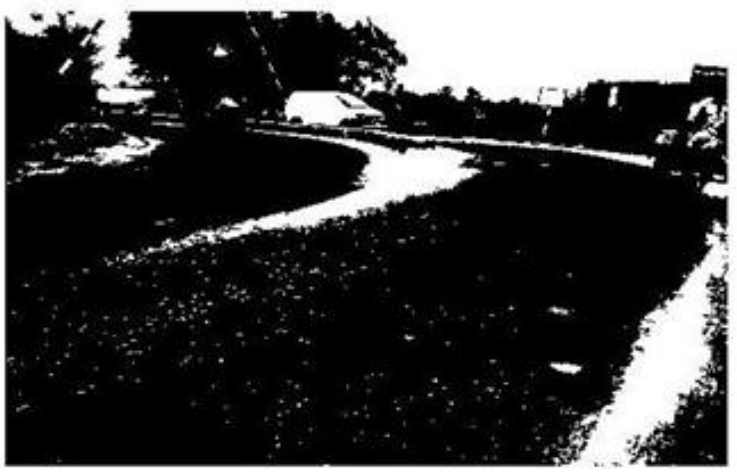

Fig 15: Region Based Segmentation
4.4 Water shade segmentation

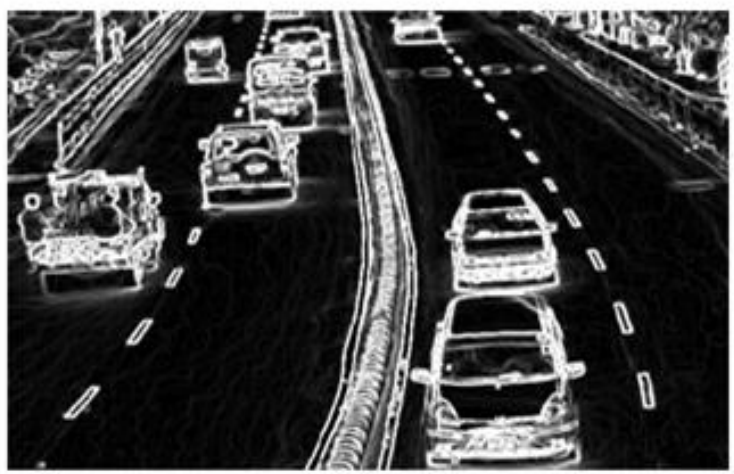

Figl 6: Water Shade Segmentation

\section{CONCLUSIONS}

These first results need further validation. The biggest concern on applying these image processing algorithms to video processing is time and space. In case of real time video the environment changes with time and so does the image properties. As a result the video outputs also differ with time. Moreover, these algorithms are based upon gray scale images. So, in order to get colored outputs, different algorithms are needed. But that will increase the processing time. However, this work can be implemented in vehicles to improve the road safety. Furthermore, it can also be implemented in surveillance cameras for monitoring purposes

\section{Acknowledgements}

The author would like to express his appreciation to Hasan Mehedi and Saha Sudeb for providing valuable inputs on this work. The author is, of course, responsible for the contents.

\section{REFERENCES}

[1] http://www.who.int/violence_injury_prevention/publications/road_traffic/world_report/en/index.html

[2] Bolen $\mathrm{J}$ et al. Overview of efforts to prevent motor vehicle-related injury. In: Bolen J, Sleet DA, Johnson V, eds. Prevention of motor vehicle-related injuries: a compendium of articles from the Morbidity and Mortality Weekly Report, 1985-1996. Atlanta, GA, Centers for Disease Control and Prevention, 1997..

[3] http://www.wisegeek.com/what-is-video-processing.htm

[4] Jones, W. ,Keeping cars from crashing, IEEE Spectrum, Vol. 38, No. 9, pp. 40-45. (2001):

[5] Rafael C. Gonzalez, Richard E. Woods ,Digital image processing Second Edition

[6] http://www.oculist.net/downaton502/prof/ebook/duanes/pages/v8/v8c016.html

[7] http://homepages.inf.ed.ac.uk/rbf/HIPR2/pixlog.htm.

[8] Oge Marques, Practical Image and Video Processing using Matlab,

[9] Birkhauser, Boston, Jean-Michel Morel and Sergio Solimini, Variational methods in Image Segmentation , Progress in Nonlinear Differential Equations and Their Applications, Vol 14

[10] POLAK, M., ZHANG, H. \& PI, M.,An evaluation metric for image segmentation of multiple objects. Image and Vision Computing, 27(8):1223-1227. 2009.

[11] UDUPA, J. K., LEBLANC, V. R., ZHUGE, Y., IMIELINSKA,C.,SCHMIDT,H., CURRIE,L.M.,et,,A framework for evaluating image segmentation algorithms. Computerized Medical Imaging and Graphics, 30( ):75-87. al. 2006.

[12] J., MUNOZ, X., RABA, D., MARTI, J. \& CUFI ,Yet Another Survey on Image Segmentation: Region and Boundary Information Integration. Springer:408 - 422 FREIXENET , X. 2002.

[13] CHAN, T. F. \& VESE, Active Contours Without Edges. IEEE, 10(2):266-277., L. A. 2001

[14] CHERIET, M., SAID, J. N. \& SUEN,A Recursive Thresholding Technique for Image Segmentation. IEEE, 7(6). C.

[15] DAWOUD, A. \& KAMEL, Iterative Multimodel Subimage Binarization for Handwritten Character Segmentation. IEEE, 13(9):1223-1230. M. S. 2004.

[16] REKIK, A., ZRIBI, M., HAMIDA, A. B. \& BENJELLOUN, An Optimal Unsupervised Satellite image Segmentation Approach Based on Pearson System and k- Means Clustering Algorithm Initialization. Internationaal Journal of Signal Processing, 5(1). M. 2009.

[17] Jos B.T.M. Roerdink and Arnold Meijster ,The Watershed Transform: Definitions, Algorithms and Parallelization Strategies; Fundamenta Informaticae 41 (2001) 187-228; IOS Press. ; 